\title{
Maintenance of pronation function after pronator teres to flexor pollicis longus tendon transfer: a cadaver study
}

\author{
Nathan P. Jarrett $\mathbb{B}^{1} \cdot$ Gregory A. Merrell ${ }^{2}$ \\ Received: 28 April 2019 / Revised: 2 September 2019 / Accepted: 3 September 2019 / Published online: 18 September 2019 \\ (c) The Author(s), under exclusive licence to International Spinal Cord Society 2019
}

\begin{abstract}
Study design Mechanistic cadaver study.

Objectives Patients in groups 4 and 5 of the International Classification for Surgery of the Hand in Tetraplegia have a few options for tendon transfer donors, but in general the needs for reestablishing motor power exceed the donor options, such that any increase in the number of potential transfers can enhance function. Although transfer of brachioradialis (BR) in these patients is well-established, pronator teres (PT) may also be a suitable donor due to its strength and excursion. It has not been extensively studied in this role, possibly because of concerns about its expendability as the major agonist of forearm pronation. The purpose of this study is to quantify forearm pronation capability pre- and post-tendon transfer of PT to flexor pollicis longus (FPL) in a cadaver model.

Setting Surgery center in Indianapolis, USA.

Methods Five cadaver arms were evaluated for pronation capability against gravity before and after PT to FPL tendon transfer. In both stages, the arms were also assessed for the pronation forces produced at the wrist when pulling PT with 25 , 50 , and $75 \mathrm{~N}$ of force. With each force, the arms were tested in full supination and neutral position.

Results All five arms were capable of pronating against gravity before and after the PT to FPL tendon transfer. Following the transfer, pronation force at the wrist decreased by $1-5 \mathrm{~N}$, but the change was not statistically significant.

Conclusions PT to FPL tendon transfer produces thumb flexion while retaining the forearm's ability to pronate and would likely retain good clinical function, freeing up BR to recreate active finger flexion or extension.
\end{abstract}

\section{Introduction}

A few tendon transfer donor options exist for groups 4 and 5 of the International Classification for Surgery of the Hand in Tetraplegia, but in general the needs for reestablishing motor power exceed the donor options, such that any increase in the number of potential transfers can enhance function [1]. Patients within these two groups present with a spinal cord injury below C6, which eliminates finger flexion and extension. While the use of brachioradialis (BR) in tendon transfers is well-established, pronator teres (PT) is

Gregory A. Merrell

gregmerrell@gmail.com

1 Medical Student, Indiana University School of Medicine, Indianapolis, IN, USA

2 Attending Surgeon, Indiana Hand to Shoulder Center, Indianapolis, IN, USA also functional in these patients; like BR, it may be a suitable donor, due to its great strength and reasonable excursion. By utilizing PT, BR is free to recreate active finger or thumb extension via transfer to extensor digitorum communis and extensor pollicis longus, respectively, or active finger flexion by transfer to flexor digitorum profundus [2, 3]. Out of all the muscles in the forearm, PT is the greatest contributor to pronation. Thus, its use as a donor tendon may be limited due to concern for its expendability. This paper is an effort to look at if it can be sacrificed, in other words, can the forearm still pronate even with PT transferred.

According to computer modeling, forearm pronation produces just under $10 \mathrm{Nm}$ of torque; the PT moment contribution is roughly $4.3 \mathrm{Nm}$, while the remaining torque is supplied by six other muscles (pronator quadratus, flexor carpi radialis, flexor digitorum superficialis, abductor pollicis longus, extensor carpi radialis longus, palmaris longus) [4]. In tetraplegia groups 4 and 5, the pronator quadratus, flexor digitorum superficialis, abductor pollicis longus, and 
palmaris longus are nonfunctional. The extensor carpi radialis longus is functional but is typically used in the tendon transfer reconstruction of finger flexion, serving as a donor to flexor digitorum profundus. This would leave only the flexor carpi radialis in group 5 patients in addition to the PT in groups 4 and 5 patients as structures contributing to pronation. This model showed PT can exert a maximum isometric force of 377 N [4]. During contraction, PT has an excursion of at least $3.3 \mathrm{~cm}$ [5]. For comparison, BR can be mobilized $6.1 \mathrm{~cm}$ on average during tetraplegia reconstructions, although the predicted active tendon tension of a BR to flexor pollicis longus (FPL) transfer is only $40 \mathrm{~N}$ $[6,7]$. Based on these factors, tendon transfer of PT to a muscle such as FPL, which has an excursion of $5 \mathrm{~cm}$ and contraction force of up to $120 \mathrm{~N}$, are promising in their potential $[8,9]$. A 2014 study found tendon transfer from PT to FPL to be equally efficacious as BR to FPL in recreating key pinch for three tetraplegic patients, although it did not evaluate the residual pronation ability [10].

The purpose of this study is to quantify pronation force capabilities pre- and post-tendon transfer of PT to FPL in a cadaver model. We hypothesize that a PT to FPL transfer will preserve the forearm's ability to pronate against gravity. The primary outcome of this study is to determine the ability of pronation against gravity after transfer of the PT to FPL. Secondary outcome measures are to identify the potential change in pronation force after PT to FPL transfer quantifiably demonstrating the usefulness of PT as an additional motor unit for hand surgery in tetraplegic patients.

\section{Methods}

A total of five left and right cadaver arms $(n=2$ left and $n=3$ right; average age, 72.6 years) composed the pronation study model. The donor weights at time of death ranged from 40 to $130 \mathrm{~kg}$, providing a variety of body types. A rig was designed to mount the prepared arms for force testing (Fig. 1). Two electronic force gauges measured the contraction-mimicking pull on PT and the resulting pronation force at the wrist.

The anterior (Henry) approach exposed the volar aspect of the superficial muscle bellies from the distal third of the forearm to the elbow proximally. PT was freed from proximal adhesions (up to $3 \mathrm{~cm}$ from the proximal attachment) allowed for greater excursion of the muscle during testing. Braided polyester \#3 suture provided a pull string after being secured to the PT tendon. A loop of \#1 suture secured to strong fascial/retinacular tissue at the radial aspect of the wrist served as an attachment point for the force gauge that would measure pronation force. A $5 / 8^{\prime \prime}$ drill bit was used to bore a transverse hole through the humerus perpendicular to its long axis, roughly $5 \mathrm{~cm}$ proximal to the elbow. Since a patient would typically maintain wrist extension when firing thumb flexion,

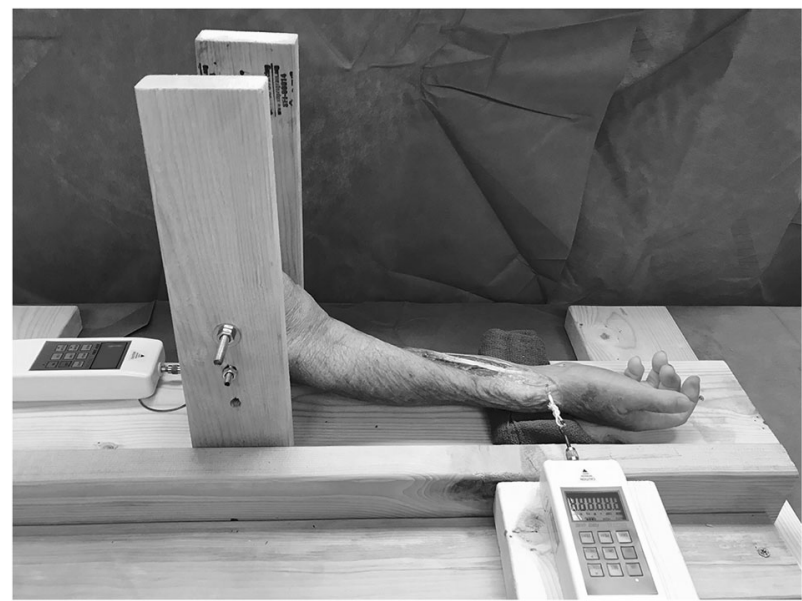

Fig. 1 Experimental setup with forearm in the fully supinated position. The proximal (left) and distal (right) force gauges recorded the peak pull force and pronation force, respectively

we locked the wrist in $30^{\circ}$ extension with two $1.3 \mathrm{~mm} \mathrm{~K}$ wires driven dorsally between metacarpals II, III, and IV into the distal radius. Fluoroscopy confirmed the K-wire placement. Measurement of the pretransfer pronation forces followed these preparations.

To perform the transfer, the distal tendinous attachment of PT and some Sharpey's fibers were dissected off of the radius. After making a short longitudinal incision through the FPL tendon, the free PT tendon was pulled through the incision, creating at least $5 \mathrm{~cm}$ of overlap [2]. Two clamps grasped the distal end of PT and proximal FPL and were pulled to overlap the tendons, creating firm thumb grasp pressure at a tension consistent with a tetraplegic tendon transfer. Using the locked loops of the Krackow technique of tendon repair, the PT and FPL were secured with \#1 suture along their tendons to complete the transfer and create a pull string from this same suture, allowing for simulated contraction of PT [11]. Larger caliber suture was used in the transfer than is typical since we were attempting to model the pronation strength of a healed transfer and did not want any failure of the transfer site itself.

Positioned between the rig's vertical posts, the arm was mounted with one bolt through the humerus and one anterior to it to prevent elbow flexion, mimicking the clinical setting in which patients have native or reconstructed elbow control (Fig. 2). Prior to collection of the wrist pronation force data, each native and transferred forearm was tested for its ability to pronate against gravity. The suture pull string simulated active contraction of PT, in line with the origin of the PT. While pulling on the suture pull string, we recorded the force required to pronate the forearm from full supination to neutral and then from neutral to full pronation.

The remaining study parameters were the pull force on PT and resulting force of pronation at the wrist. Having attached the distal force sensor to the wrist suture and the PT pull 


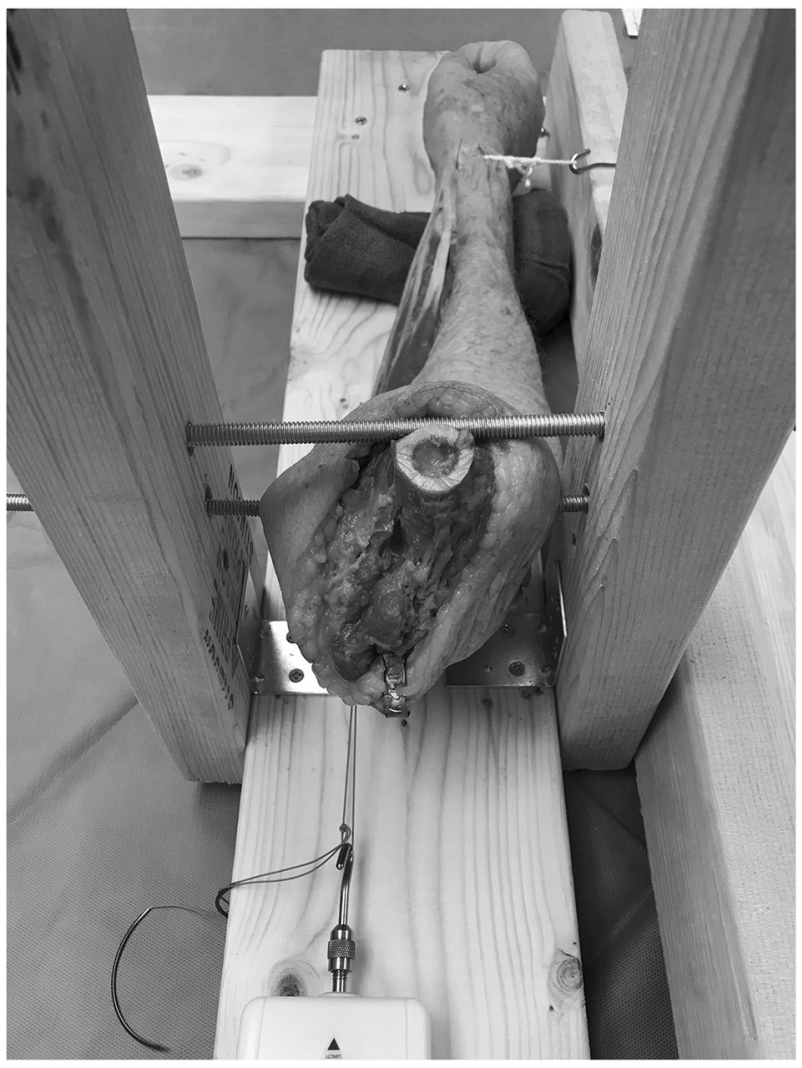

Fig. 2 Experimental setup with the forearm in neutral position. The arms were mounted by a rod through the humerus, while a second, anterior bolt kept the elbow from flexing while PT was pulled

string suture to the proximal force sensor, the forearm was tested in full supination and in the neutral position. We pulled the proximal force sensor to supply $25 \mathrm{~N}$ of pull force to $\mathrm{PT}$ and recorded peak pronation force at the wrist. Three trials of each pull force-25, 50, $75 \mathrm{~N}$-were recorded in both arm positions. Once the tendon transfer was complete, all measurements were repeated, including pronation against gravity.

In a few instances, the transfer decreased the force required to pronate against gravity; thus, we ran a two-tailed paired $t$ test to compare the threshold force for pronation against gravity in native and transferred arms. We also ran a two-tailed paired $t$ test to compare the pre- and post-transfer pronation data and determine if the post-transfer force was significantly lower than the native pronation force. $P \leq 0.05$ was considered significant.

\section{Results}

All five arms were capable of pronation against gravity before and after the tendon transfer. Depending on the arm position, the minimum required force ranged from 12 to $34 \mathrm{~N}$ for native arms and $10-39 \mathrm{~N}$ for transferred arms (Table 1A). There was no significant difference between pre- and post-transfer pronation from full supination to neutral, but the neutral to full pronation force shows a statistically significant increase after the transfer $(26-30 \mathrm{~N} ; P \leq 0.05)$ (Table $1 \mathrm{~A})$.

Upon measuring pronation forces at the wrist, post-transfer forearms produced less force in both positions tested, and the neutral positions generated the most force in their respective transfer status groups (Table 1B, Fig. 3). In the fully supinated position, the transfer decreased pronation force by $15-28 \%$; in the neutral position, force decreased by $23-35 \%$ (Table 1C). However, a two-tailed paired $t$ test showed that there was no statistically significant difference between the pronation force before and after the transfer.

\section{Discussion}

This study met the primary outcome measure demonstrating that PT is an effective pronator even after tendon transfer to FPL. Meaningful findings included the ability of all tested arms to "actively" pronate through use of a suture pull string before and after tendon transfer. Although there was a statistically significant difference between the pre- and posttransfer force required to pronate against gravity from the neutral position, no difference existed between the two groups when beginning in the fully supinated position. It is unclear as to why there was a difference when starting from the neutral position, but the new route of the tendons across the forearm may alter the movement mechanics, possibly through muscle length-dependent effects that only become apparent near the end of the pronation arc.

By transferring PT off of the radius and onto FPL, which has radial attachments, there is potential for some loss of pronation force, although it appears to be statistically insignificant (Table 1C). The previously cited study of PT to FPL by Mohindra et al. provides support for its efficacy in recreating key pinch. Thus, for tetraplegic patients who are willing and able to undergo reconstructive limb surgery, there is reason to believe that PT can be used as a donor motor unit due to evidence that supports restoration of thumb flexion and minimal loss of pronation.

Admittedly, the sample size of five arms poses a limitation on this study. Arms of different sizes and donor weights were tested, improving the applicability to different patient body types. The PT pull force and the resulting pronation force are intimately associated with the forearm's properties, which led to a wide range of forces required to pronate against gravity (Table 1A).

At the very least, patients receiving this treatment should be able to pronate their forearm against gravity while regaining some of their thumb flexion. Although the postoperative force of pronation may not be comparable with that of a healthy individual without spinal cord injury, grasp and pinch functions are absent in groups 4 and 5 tetraplegic 
Table 1 Threshold of Force Required to Pronate Against Gravity, Average Pronation Force Generated, and Percentage Decrease in Average Pronation Force Post-Transfer. All measures of variability shown are standard error (SE)

\begin{tabular}{|c|c|c|c|c|}
\hline & \multicolumn{2}{|l|}{ Native } & \multicolumn{2}{|l|}{ Transferred } \\
\hline & Full supination to neutral & Neutral to full pronation & Full supination to neutral & Neutral to full pronation \\
\hline \multicolumn{5}{|c|}{ A. Threshold of force required to pronate against gravity $(N)$} \\
\hline Highest & 23.3 & 33.5 & 32.6 & 38.9 \\
\hline Lowest & 12.4 & 16.5 & 9.5 & 21.5 \\
\hline Average for five arms & $19(2.1)$ & $25.6(4.3) *$ & $24.5(2.9)$ & $30.3(3.3)^{*}$ \\
\hline \multicolumn{5}{|c|}{ B. Average pronation force generated $(N)$} \\
\hline \multicolumn{5}{|l|}{ PT pull force } \\
\hline 25 & $3.9(0.5)$ & $4.4(0.4)$ & $3.3(0.3)$ & $3.4(0.6)$ \\
\hline 50 & $7.1(0.6)$ & $9.8(0.4)$ & $5.9(0.3)$ & $6.4(0.6)$ \\
\hline 75 & $10.7(1.0)$ & $14.7(0.8)$ & $7.7(0.9)$ & $9.5(0.5)$ \\
\hline \multicolumn{5}{|c|}{ C. Percentage decrease in average pronation force post transfer $(\%)$} \\
\hline \multicolumn{5}{|l|}{ PT pull force $(N)$} \\
\hline 25 & 15 & 23 & & \\
\hline 50 & 17 & 35 & & \\
\hline 75 & 28 & 35 & & \\
\hline
\end{tabular}

*Statistically significant difference found between the native and transferred models' threshold of force required to pronate against gravity from neutral to full pronation

Fig. 3 The average pronation forces corresponding with each pull force. Pronation from the neutral position prior to transfer produced the greatest force, but there was no statistically significant difference between the pre- and post-transfer forces when comparing the same forearm positions. The data points in the figure were offset around each pull force to avoid overlap of the standard error (SE) bars

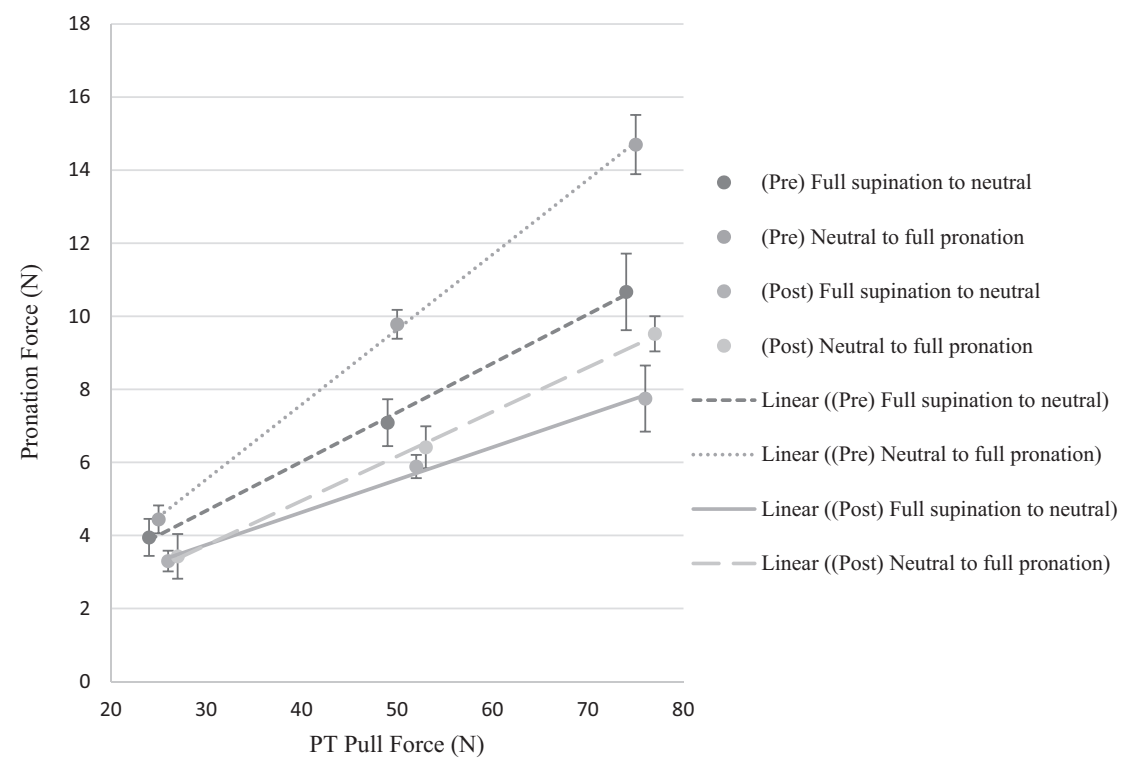

patients who do not receive reconstructive limb surgery. Post transfer, the positioning of PT still allows it to contribute to pronation. Thus, having both the ability to pronate against gravity at a minimum and an additional tendon donor suitable for transfer creates further options for restoring hand function.

By demonstrating through a cadaver model that the use of PT as a donor to FPL does not reduce pronation capability to a statistically significant extent, we believe this will be translatable to a clinical context and can provide surgeons with an additional motor unit for treatment of tetraplegic patients. Being a cadaver study, this work is a starting point for future investigation, providing a quantified observation of the pronation force following PT to FPL transfer. Future work is necessary to validate the feasibility of this procedure in vivo; next steps might be experimental trials in patients who are good candidates for multi-stage reconstruction that incorporates BR transfer to other finger flexors or extensors.

\section{Data availability}

The datasets generated during and/or analyzed during the current study are available in the Mendeley repository, https://doi.org/10.17632/67m827f74d.1\#file-5373df349711-4b73-addb-ed92719a11a5. 
Acknowledgements The authors would like to acknowledge Zach Bolinger for his help with preparing Figs. 1 and 2 for publication and George Eckert for his technical assistance with Fig. 3.

Funding No financial assistance was received in support of the study.

Author contributions NPJ contributed to study design and execution, data analysis and interpretation, and was responsible for constructing the model, writing the paper, and preparing the data tables. GAM contributed to study design and execution, data analysis and interpretation, and provided feedback on the paper editing.

\section{Compliance with ethical standards}

Conflict of interest The authors declare that they have no conflict of interest.

Ethical approval We certify that all applicable institutional and governmental regulations concerning the ethical use of human specimens were followed during the course of this research.

Publisher's note Springer Nature remains neutral with regard to jurisdictional claims in published maps and institutional affiliations.

\section{References}

1. Fridén J, Gohritz A. Tetraplegia Management Update. J Hand Surg Am. 2015;40:2489-500.
2. Van Heest, AE Tetraplegia. In: Wolfe SW, Hotchkiss RN, Pederson WC, Kozin SH, Cohen MS, editors. Green's Operative Hand Surgery. 7th edn. Philadelphia, Elsevier, 2017: p. $1122-145$.

3. Srikanth R, Rayid K, Kakumanu S. Brachioradialis to flexor digitorum profundus tendon transfer to restore finger flexion. $\mathrm{J}$ Plast Surg. 2018;51:123

4. Hale R, Dorman D, Gonzalez RV. Individual muscle force parameters and fiber operating ranges for elbow flexion-extension and forearm pronation-supination. J Biomech. 2011;44:650-6.

5. Abrams GD, Ward SR, Fridén J, Lieber RL. Pronator teres is an appropriate donor muscle for restoration of wrist and thumb extension. J Hand Surg Am. 2005;30:1068-73.

6. Kozin SH, Bednar M. In vivo determination of available brachioradialis excursion during tetraplegia reconstruction. J Hand Surg. 2001;26:510-4.

7. Fridén J, Shillito MC, Chehab EF, Finneran JJ, Ward SR, Lieber RL. Mechanical feasibility of immediate mobilization of the brachioradialis muscle after tendon transfer. J Hand Surg. 2010;35:1473-8.

8. Trumble TE, Sailer SM, Gilbert MM. Principles of Hand Surgery and Therapy. Philadelphia: W.B. Saunders; 2000. p. 343.

9. An KN, Cooney WP, Chao EY, Askew J, Daube JR. Determination of forces in extensor pollicis longus and flexor pollicis longus of the thumb. J Appl Physiol Respir Environ Exerc Physiol. 1983;54:714-19.

10. Mohindra M, Sangwan SS, Kundu ZS, Gogna P, Tiwari A, Thora A. Surgical Rehabilitation of a Tetraplegic Hand: Comparison of Various Methods of Reconstructing an Absent Pinch and Hook. Hand. 2014;9:179-86.

11. Fitoussi F, Bachy M. Tendon lengthening and transfer. Orthop Traumatology: Surg Res. 2015;101:S149-57. 